\title{
A Comprehensive Model for Measuring Information System Performance for Treating Chronic Adhesive Capsulitis
}

\author{
Seong-Ran Lee \\ Department of Medical Information, Kongju National University \\ leesr@kongju.ac.kr
}

\begin{abstract}
This paper is to develop a comprehensive model for measuring information system performance for treating chronic adhesive capsulitis. The data were collected by interview and questionnaires from August 10 to September 25, 2015. The $X^{2}$-test was used to observe a statistically significant difference of general characteristics between experimental and control group. As a result, firstly, for marital status, the percent of single $(21.3 \%)$ in the experimental group were significantly a lower rate than the proportion (36.0\%) of single in the control group $\left(X^{2}=3.41, p<0.05\right)$. Secondly, for the effectiveness of massage therapy, respondents' score (32.19 \pm 0.57$)$ after application significantly increased than respondents $(17.59 \pm 1.55)$ before application of a comprehensive model $(t=-0.16, p=0.00)$. Thirdly, for health practice, the result of follow-up survey in patients with adhesive capsulitis of shoulder, the mean scores of health practice showed an increase in the experimental group, regardless of the time elapsed of 60 days after the information application. Therefore, the comprehensive model of this system will contribute to reducing chronic adhesive capsulitis of shoulder in the future.
\end{abstract}

Keywords: Comprehensive model, Information system, Performance, Adhesive capsulitis, Self-activities

\section{Introduction}

Adhesive capsulitis of shoulder also known as frozen shoulder is a painful and disabling condition of unclear part in which the upper limb. The bone and muscle tissue surrounding the aponeurosis of the shoulder becomes inflamed and hard, greatly restricting movement and causing chronic work [1-3]. Pain is usually continuity, worse at evening, and with winter temperature. Certain movements or state can present condition of tremendous pain and cramping. Adhesive capsulitis occurs well after the 50-year-old women. Particulary, it often occurs shoulders aching with the disease. It was characterized by a hard movement from the 60 to 80 years old that occurs in 3-7\% is a common disease [3-5]. Movement of the shoulder is severely limited, with pain loss of both shoulder and passive limitation of movement ${ }^{[1]}$ The part is sometimes caused by disorder, leading to lack of movement due to pain and stiffness of shoulder but also often arises spontaneously with no obvious trigger element. Rheumatis and surgery of shoulder joint can also cause a condition of pain and limitation of movement similar to adhesive capsulitis. periods of use may cause inflammation [6-7].

In adhesive capsulitis, there is a lack of synovial fluid, which normally helps the shoulder bone, a ball and socket bone, move by lubricating the gap between the upper limbs and the socket in the shoulder blade. The shoulder capsule thickens, swells, and tightens due to bands of scar part that have made inside the ligament. As a result, there is less room in the bone for the upper limbs, making movement of the shoulder limitation. This restricted space between the capsule and ball of the upper limb distinguishes adhesive capsulitis from a less complicated, painful, stiff shoulder [5, 8]. 
The incidence of adhesive capsulitis is about 5 percent in the general population. The incidence of adhesive capsulitis is about 5 percent in the general population. Occurrence is rare in children and people under 30 but peaks between 50 and 60 years of age. Women are more often influenced than men and it is common in patients with diabetes mellitus. The incidence of adhesive capsulitis is about 3 percent in the general population. At least in its idiopathic form, the condition is much more common in women than in men. The disease presents approximately $70 \%$ of patients who are women aged 40-60. Adhesive capsulitis is more frequent in diabetic patients and is more symptoms and more disorder than in the non-diabetic population. Recent vaccine-related shoulder disorder are increasing in patients with women $[9,10]$.

People with diabetes mellitus, cerebrovascular accident, pneumothorax, degenerative arthritis, or angina pectoris are at a higher risk for frozen shoulder. Injury or surgery to the shoulder or upper limb may cause blood flow damage or the capsule to tighten from reduced use during recovery. Adhesive capsulitis has been indicated as a possible adverse effect of some forms of highly active muscle therapy. Some cases have also been reported after mastectomy and lung surgery. Surgery is recommended nonsurgical treatment is not effective. But there are complications. That is stiffness and pain continue even with therapy. The condition can lead to depression, pain, disorder in the cervical part and back and severe weight loss due to long-term lack of deep sleep. People who suffer from adhesive capsulitis may have extreme difficulty concentrating, working or performing daily life activities for extended periods of time [11, 12].

In order to treatment the disorder, we should look for practical and useful contents. However, there were few researches to deal with contents of information system for the treatment in patients with adhesive capsulitis so far.

Therefore, a comprehensive model of adhesive capsulitis is needed to control the increasing prevalence of adhesive capsulitis and conduct its related desirable outcomes. Thus, this paper is to estimate a comprehensive model of an information system for treating chronic adhesive capsulitis. That is, the paper is to develop an information system for improving adhesive capsulitis. A comprehensive and systematic adoption of an information system to minimize the damage of adhesive capsulitis of shoulder will contribute more to the rapid disorder loss and discomfortable function.

\section{Materials and Methods}

\subsection{Study Materials}

The subjects of this study were 150 persons who have visited in orthopedic surgery at a general hospital in Metropolitan province which was willing to participate in the program. The subjects were randomly classified into two groups of each part 75 . One group was for the experimental group and the other group was for the control group. The experimental group of 75 patients which was assigned as part by application of a comprehensive model, while the control group of 75 patients was assigned as part without application of a comprehensive model.

\subsection{Contents of A Comprehensive Model}

The contents of a comprehensive model are as follows. Skill, motivation, timeliness, impact, improvement and others in Figure 1. For detailed contents, there are recognition, attitude, accurate, improvement. In addition, time assigned for patients with frozen shoulder is as follows. Discussion, teaching, efficiency, evaluation and others. There were accurate entry of patient status and therapeutic procedure and evaluation of information system quality in Figure 2-3. 


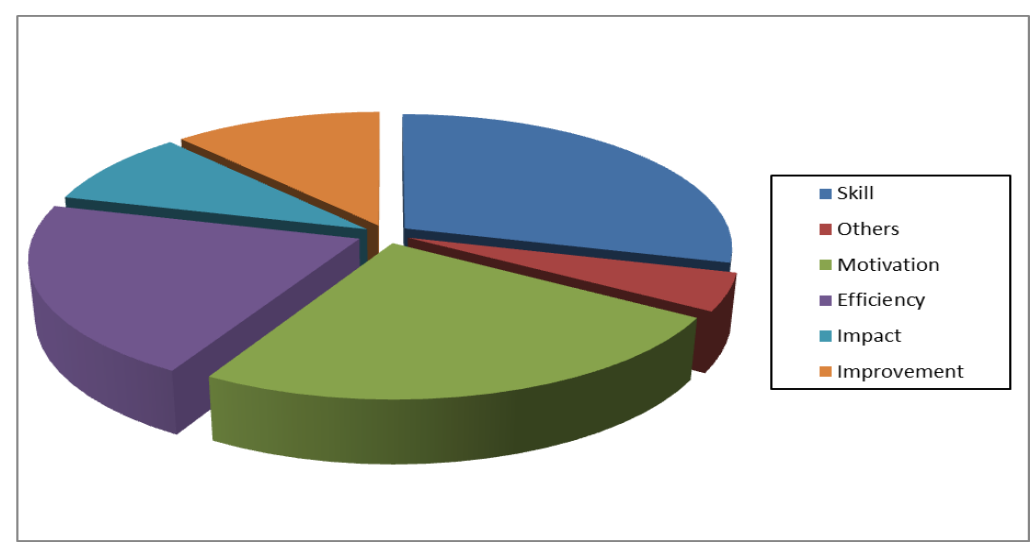

Figure 1. Contents of A Comprehensive Model

\subsection{Study Methods}

The $\mathrm{X}^{2}$-test was used to observe a statistically significant difference of general characteristics between experimental and control group. The pairwise t-test was done to compare the change of self-activities before and after application of a comprehensive model. The t-test was also performed to determine a statistically significant difference between the two groups on user satisfaction of the information system for measurement of activities of daily living for the prevention of recurrence in adhesive capsulitis.

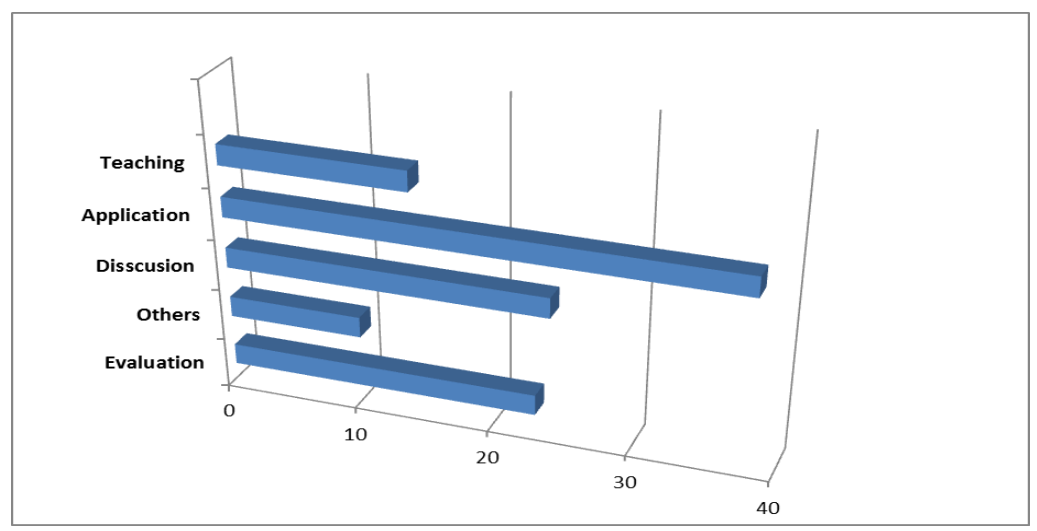

Figure 2. Time Assigned for Patients with Chronic Adhesive Capsulitis 
- Optimized design system

- Identifying a problem through related model

- Need assessment of the participants

- Cord, input, file, process, and output system

- Identifying the functional elements of successful models

- Program formation about clinical information

- Designing as part of information gathering and synthesis

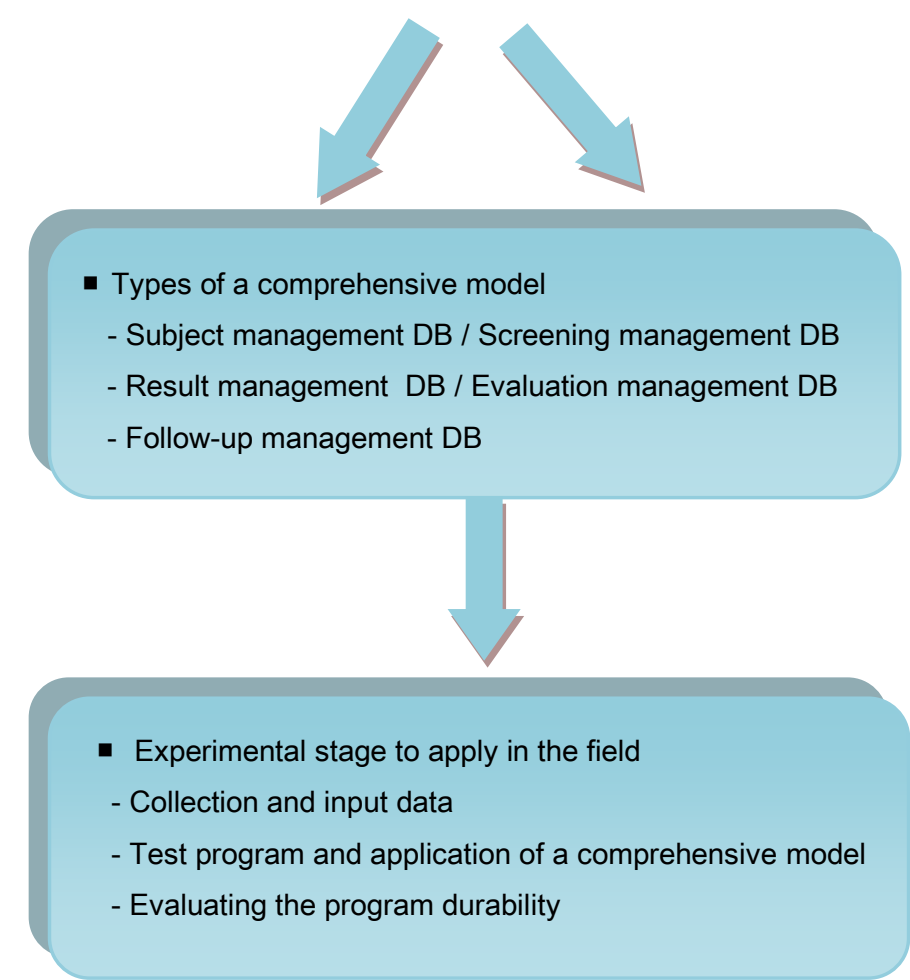

Figure 3. A Comprehensive Model for Patients with Chronic Adhesive Capsulitis

\section{Results}

\subsection{Basic Information of Subjects in This Study}

Table 1 presents basic information of subjects in this study. The subject's gender was analyzed. Male with $54.7 \%$ in the experimental group showed more than female with $52.0 \%$ in the control group. Female with $45.3 \%$ in the experimental group showed a lower rate than female with $48.0 \%$ in the control group. In the other hand, for marital 
status, the percent of single $(21.3 \%)$ in the experimental group were significantly a lower rate than the proportion $(36.0 \%)$ of single in the control group $\left(\mathrm{X}^{2}=3.41, \mathrm{p}<.05\right)$.

Table 1. General Characteristics of Subjects in This Study

\begin{tabular}{|c|c|c|c|}
\hline \multirow[b]{2}{*}{ Variables } & Experi & Cont & \multirow[b]{2}{*}{$X^{2}$} \\
\hline & $\mathrm{N}(\%)$ & $\mathrm{N}(\%)$ & \\
\hline \multicolumn{4}{|l|}{ Gender } \\
\hline Male & $41(54.7)$ & $39(52.0)$ & 9.28 \\
\hline Female & $34(45.3)$ & $36(48.0)$ & \\
\hline \multicolumn{4}{|l|}{ Age } \\
\hline$\leq 39$ & $7(9.3)$ & $10(13.3)$ & 10.37 \\
\hline $40-49$ & $15(20.0)$ & $18(24.0)$ & \\
\hline $50-59$ & $30(40.0)$ & $21(28.0)$ & \\
\hline$\geq 60$ & $23(30.7)$ & $26(34.7)$ & \\
\hline \multicolumn{4}{|l|}{ Monthly income } \\
\hline$\leq 99$ & $16(21.3)$ & $10(13.3)$ & 5.62 \\
\hline $100-200$ & $11(14.7)$ & $17(22.7)$ & \\
\hline 201-299 & $27(36.0)$ & $30(40.0)$ & \\
\hline$\geq 300$ & $21(28.0)$ & $18(24.0)$ & \\
\hline \multicolumn{4}{|l|}{ Marital status } \\
\hline Single & $16(21.3)$ & $27(36.0)$ & $3.41^{*}$ \\
\hline Married & $59(78.7)$ & $48(64.0)$ & \\
\hline \multicolumn{4}{|l|}{ Education level } \\
\hline Under middle & $19(25.3)$ & $22(29.3)$ & 10.6 \\
\hline High school & $25(33.3)$ & $34(45.3)$. & \\
\hline Over college & $31(41.3)$ & $19(25.3)$ & \\
\hline Total & $75(100.0)$ & $75(00.0)$ & \\
\hline
\end{tabular}

\subsection{Comparison of Self-Activities After Application of a Comprehensive Model}

Table 2 represents the comparison of self-activities after application of a comprehensive model. For the change of shoulder pain, respondents' score $(24.63 \pm 0.79)$

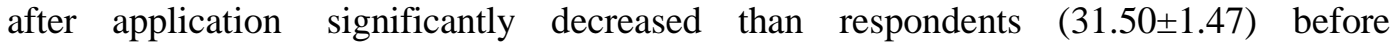
application $(\mathrm{t}=0.48, \mathrm{p}=0.00)$. For physical function, respondents' score $(32.19 \pm 0.57)$ after application significantly increased than respondents $(17.59 \pm 1.55)$ before respondents $(\mathrm{t}=-0.16, \mathrm{p}=0.00)$ after the massage. 
Table 2. Comparison of Self-Activities After Application of a Comprehensive Model

\begin{tabular}{|c|c|c|c|c|}
\hline & Before & After & & \\
\hline & Mean \pm S.D & Mean \pm S.D & $\mathrm{t}$ & $\mathrm{P}$ \\
\hline Physical function & & & & \\
\hline $\begin{array}{l}\text { Physical symptoms } \\
\text { stiffness }\end{array}$ & $34.97 \pm 0.62$ & $28.14 \pm 1.52$ & 2.06 & 0.00 \\
\hline Shoulder pain & $31.50 \pm 1.47$ & $24.63 \pm 0.79$ & 0.48 & 0.00 \\
\hline Limitation of & $33.64 \pm 0.83$ & $21.96 \pm 2.51$ & 1.62 & 0.00 \\
\hline Movement & & & & \\
\hline Stress & $26.81 \pm 0.71$ & $25.57 \pm 0.32$ & 1.37 & 0.42 \\
\hline Massage & $17.59 \pm 1.55$ & $32.19 \pm 0.57$ & -0.16 & 0.00 \\
\hline Exercise & $19.59 \pm 1.40$ & $23.27 \pm 1.39$ & -0.42 & 0.38 \\
\hline Compress & $11.62 \pm 0.39$ & $30.15 \pm 0.82$ & -0.75 & 0.00 \\
\hline Dietary practice & & & & \\
\hline Onion intake & $24.63 \pm 1.82$ & $27.60 \pm 1.48$ & -2.74 & 0.52 \\
\hline Anchovy intake & $25.84 \pm 0.36$ & $32.17 \pm 0.85$ & -1.47 & 0.00 \\
\hline Milk intake & $16.36 \pm 1.85$ & $19.52 \pm 1.29$ & -0.28 & 0.49 \\
\hline Almond & $9.72 \pm 0.72$ & $12.49 \pm 0.51$ & -1.62 & 0.37 \\
\hline Clinical condition & & & & \\
\hline Sea mustard & $26.95 \pm 0.34$ & $33.71 \pm 0.39$ & -1.39 & 0.00 \\
\hline Smoking & $22.62 \pm 1.59$ & $21.62 \pm 1.46$ & 3.74 & 0.59 \\
\hline Alcohol drinking & $25.18 \pm 0.86$ & $22.17 \pm 1.72$ & 1.59 & 0.46 \\
\hline Diabetes mellitus & $22.57 \pm 1.36$ & $20.28 \pm 1.35$ & 2.82 & 0.41 \\
\hline Hypertension & $26.48 \pm 0.73$ & $19.62 \pm 0.59$ & 0.74 & 0.00 \\
\hline Cholesterol & $26.75 \pm 1.29$ & $22.49 \pm 1.67$ & 0.51 & 0.34 \\
\hline
\end{tabular}

\subsection{Follow-up Survey of Health-Related Lifestyle Behaviors After Application}

Figure 4-5 presents the follow-up survey of health-related lifestyle behaviors after application of the information system. For stress control, according to the health promoting behaviors after the application, the mean scores of the experimental group showed a decrease after the application than control group. On the other hand, for health practice, the result of follow-up survey in patients with adhesive capsulitis of shoulder, the mean scores of health practice showed an increase in the experimental group, regardless of the time elapsed of 60 days after the information application. 


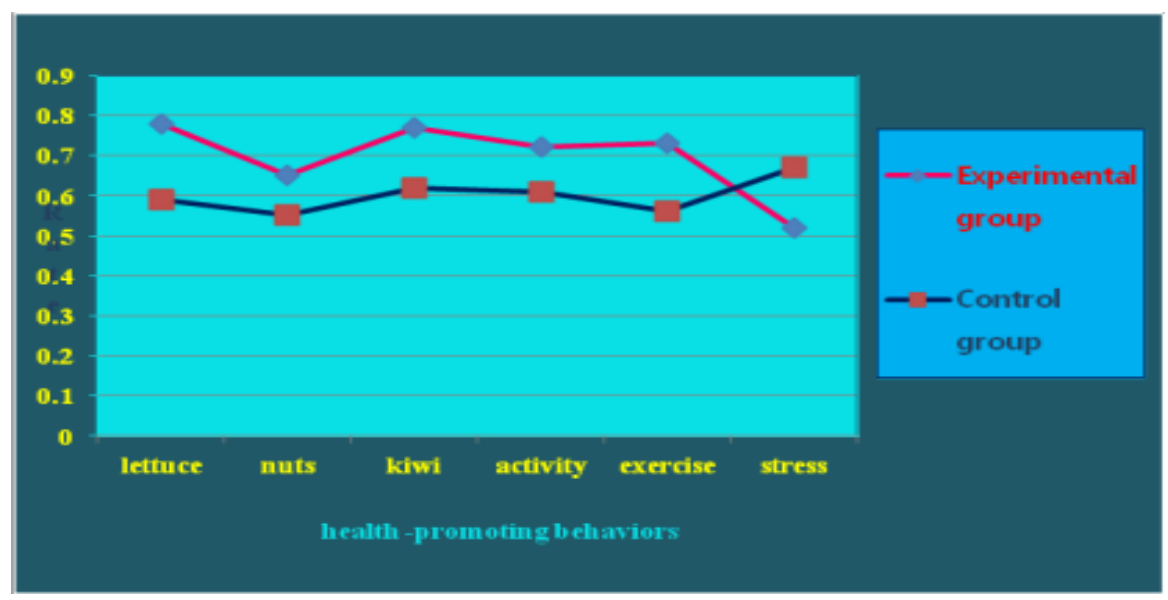

Figure 4. Follow-up Survey of Health Promoting Behaviors

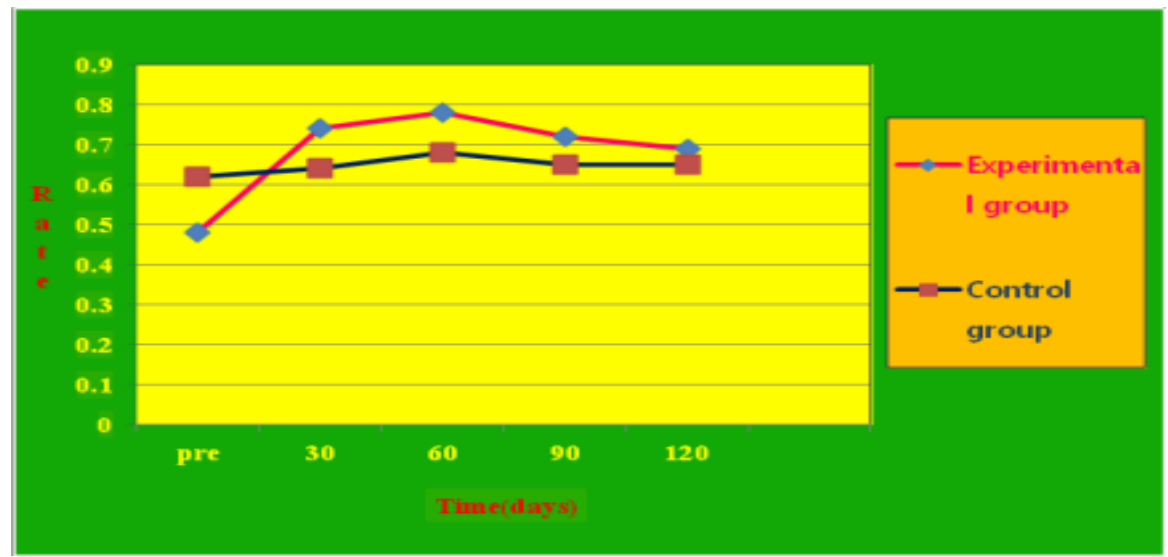

Figure 5. Follow-up Survey of Health Practice

\section{Discussion}

This paper is to estimate the effect of a comprehensive model of information systems for treating chronic adhesive capsulitis of shoulder. The application effect was significantly decreased the disorder rate of shoulder using application of the information system, and then multiple approaches is effective in reducing the adhesive capsulitis prevalence. As a result of this study, the message strategy, compression on healthy behaviors diminished the progression rate of disease. The finding was a difference to the previous studies on the frozen shoulder [13, 14]. This study suggests that patients with adhesive capsulitis of shoulder should be focused on real application testing to prevent the incidence of disease. Based on the result obtained by the research, it is anticipated that this may be used as an effective data for developing and interventing information system for the patients with adhesive capsulitis of shoulder. However, in order to prevent adhesive capsulitis, the results show that a comprehensive information is more useful than individual information program. After the experiment, the experimental group which experienced information application level on optimum information system showed the effectiveness of health practice in patients with adhesive capsulitis of shoulder. In comparison with the mean of shoulder stiffness, the experimental group's change was more positive, and there was significantly higher than control group.

As is stated above, we identified that experimental group of the application of daily activities has been effective for doing shoulder firm and pain control which alleviate the disorder of adhesive capsulitis in subjects. And the improvement was a significant 
satisfactory. The finding was similar with the results of previous researches [15],[16]. Therefore, it is believed that the application of experimental group is a unique useful program for self-improvement of subjects. And it is a new way of information system for adhesive capsulitis patients. This research will contribute to establish the advanced system and the developed structure and ultimately to improve activities of daily living in patients with adhesive capsulitis of shoulder.

A comprehensive model is any organized model for the data collection, processing, storage, analysis and communication of information. Particulary, it is the research of complementary networks that patients and diseases use to collect, analysis, filter, analysis, process, create, distribute, and evaluation data.

A comprehensive information system is a system composed of patients and contents that processes or interprets information. The term is also sometimes used in more restricted senses to refer to only the software used to run a computerized database or to refer to only a computer system. The Information model is an academic study of information systems for a specific patient to improve the diseases and the complementary networks of hardware and software. The model is an information system having a definitive boundary, users, processors, stores, inputs, outputs and the previous mentioned communication networks.

Any specific information system aims to support operations, management and decision-making. An information system is the information and communication technology (ICT) that an organization uses, and also the way in which patients interact with this technology in support of business processes. Some patients make a clear distinction between information systems, computer systems, and process. Information systems typically include an ICT component but are not purely concerned with ICT, focusing instead on the end use of information technology. The information systems are also different from other processes. The model of information systems will help to improve the performance of treatment for patients with chronic adhesive capsulitis.

\section{Conclusion}

This paper is to develop a comprehensive model for measuring information system performance for treating chronic adhesive capsulitis.

As a result, firstly, for marital status, the percent of single (21.3\%) in the experimental group were significantly a lower rate than the proportion (36.0\%) of single in the control group $\left(X^{2}=3.41, p<0.05\right)$. Secondly, for the effectiveness of massage therapy, subjects' score $(32.19 \pm 0.57)$ after application significantly increased than subjects $(17.59 \pm 1.55)$ before application of a comprehensive model $(\mathrm{t}=-0.16, \mathrm{p}=0.00)$. Thirdly, for health practice, the result of follow-up survey in patients with adhesive capsulitis of shoulder, the mean scores of health practice showed an increase in the experimental group, regardless of the time elapsed of 60 days after the information application. Therefore, the information system using intervention will contribute to reducing chronic adhesive capsulitis of shoulder in the future.

\section{Acknowledgments}

I thank them for conducting the output of research to respondents who had participated in the development of a comprehensive model for measuring information system performance for treating chronic adhesive capsulitis. This paper was a revised and expanded version of a paper entitled "Effect of A New Information Adoption for Treating Chronic Adhesive Capsulitis", Workshop on Healthcare and Nursing 2016 presented at Jeju National University International Center, April 19-21, 2016 


\section{References}

[1] S. Mahomed, T. Al-Dbaidis and B. Al-Zoabi, "Outcome Measures and Psychomotor Skills Related to Shoulder Conditions for Clinical Orthopedic Training”, Medical Princ Practice, vol. 17, no. 6, (2008), pp. 481-485.

[2] W. N. Levine, C. P. Kashyap abd S. F. Bak, "Nonoperative Management of Idiopathic Adhesive Capsulitis”, Journal Shoulder Elbow Surgical, vol. 16, no. 5, (2003), pp. 569-573.

[3] S. M. Griggs, A. Ahn and A. Green, "Idiopathic Adhesive Capsulitis, A Prospective Functional Outcome Study of Nonoperative Treatment", Journal Bone Joint Surg Am, vol. 82, no. 10, (2000), pp. 1398-1402.

[4] N. Grubbs, "Frozen Shoulder Syndrome, A Review of Life", Journal Orthop Sports Phys Ter, vol. 18, no. 3, (2013), pp. 479-482.

[5] D. Celik, "Comparison of the Outcomes of Two Different Exercise Programs on Frozen Shoulder," Acta Orthop Traumatol Turc, vol. 44, no. 40, (2010), pp. 285-285.

[6] S. Harris, SPARQI, "Query Processing with Conventional Relational Database Systems", Springer Verlag, Lecture Notes in Computer Science, LNCS, vol. 807, (2005), pp. 234-237.

[7] Jun, "Multi-granularity Locking-based Concurrency Control in Object-Oriented Database Systems", Journal of Systems and Software, vol. 54, (2000), pp. 201- 217.

[8] J. E. Shaffer and R. K. K. Tibone, "Frozen Shoulder, A Long Term Follow up", J Bone Joint Surg., vol. 74, (2002), pp. 736-738.

[9] I. Binder, "Frozen Shoulder, A Long Term Prospective Study, Ann Rheum Dis", vol. 43, (2004), pp. 361362.

[10] I. Binder, "Frozen Shoulder: An Arthrographic and Radionuclear Scan Assessment”, Ann Rheum Dis, vol. 43, (2004), pp. 365-369.

[11] T. W. Melzer, C. J. Wirth and S. Hoffmann, "Frozen Shoulder Treatment and Results", Arch Orthop Trauma Surg., vol. 1114, no. 2, (2005), pp. 87-91.

[12] R. J. Neviaser, "The Frozen Shoulder, Diagnosis and Management, Clin Orthop, vol. 223, (2007), pp. 59-64.

[13] E. A. Codman, "The Shoulder Boston Thomas Todd: Lesson Learnt From the Painful Shoulder: A Case Series of Malignant Shoulder Girdle Tumors Misdiagnosed As Frozen Shoulder", Gerald M. Y. Quan, Int emin Surg Oncol, vol. 2. no. 2, (2005).

[14] T. W. Melzer, C. Wirth and J. S. Hoffmann, "Frozen Shoulder-Treatment and Results", Arch Ort-Hop Trauma Surg., vol. 114. no. 2, (2005), pp. 87-91

[15] S. Green, R. Buchbinder and S. Hetrick, "Acupuncture for Shoulder Pain", Cochrane Database Syst Rev, vol. 8. no. 2, (2005).

[16] S. R. Lee, "Effect of A New Information Adoption for Treating Chronic Adhesive Capsulitis", Workshop on Healthcare and Nursing, Jeju Island, Korea, (2016).

\section{Author}

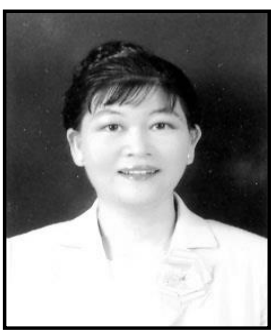

Seong-Ran Lee, she received the B.S. degree in consumer science from Seoul National University, Korea in 1987. She received the M.S. degree in health science from Seoul National University, Korea in 1992 and Ph.D in the same area from Catholic Medical College, Seoul, Korea in 2000. Currently, she is a professor in the department of medical information, Kongju National University, Korea. Her present research interest is medical information 
International Journal of Bio-Science and Bio-Technology

Vol.8, No.6 (2016) 